\title{
Infrared-based Short-Distance FSO Sensor Network System
}

\author{
https://doi.org/10.3991/ijoe.v14i12.9493 \\ Jinyong Huang, Zhe Li $\left.{ }^{(}\right)$ \\ Hubei Engineering University, Xiaogan, China \\ lizhe hbeu@vip.163.com.com
}

\begin{abstract}
Energy consumption is one of the major problems in the development of Wireless Sensor Network (WSN). Under certain application conditions, Free Space Optical (FSO) communication can effectively reduce system energy consumption. Combining the need of the short-distance communication in the cooperative control of the mobile robot, this paper focuses on the study for the infrared FSO communication method and devices. Eight infrared-transmitting and receiving modules with the angular spacing $45^{\circ}$ were deployed to realize the omnidirectional communication. The systematic measurement shows that the designed communication system can effectively carry out data transmission within a distance of 14 meters when the bit error ratio (BER) is $0 \%$. Through the link budget analysis, the theoretical transmission distance of the design system is about 24 meters, only accounting for $60 \%$ of the communication module data manual, and the BER rises rapidly as the distance increases. The analysis of the oscilloscope observation results indicates that due to the attenuation caused by each medium (mainly moisture, dust, etc.) in the propagation path, the BER rapidly increases when the distance increases. Therefore, based on the test results, this system is applicable for constructing a short-distance wireless sensor network to meet the needs of cooperative control of mobile robots, without involving the complicated pointing process.
\end{abstract}

Keywords-wireless sensor network (WSN), energy efficiency, communication design, FSO infrared.

\section{Introduction}

Energy consumption is one major issue in WSN. The energy consumption of WSN is mainly concentrated on data processing, communication and routing, and voltage and current conversion devices, among which the energy consumption of the communication part may account for $70 \%$ or more of the entire system [1] Typical WSNs generally use RF for data transmission [2], which makes their overall energy consumed mainly by these radio frequency (RF) communication modules [3]. The main reason is that RF communication is omnidirectional, and most of the energy is not effectively used in the communication between WSN nodes but consumed "insignificantly" [4-6]. Therefore, as an alternative solution, FSO communication is one of the solutions to improve energy efficiency [2-3]. 
Compared with the typical RF transmission mode, the energy consumption of the FSO mode is significantly reduced [7-8], especially when continuous communication is needed [9]. Most of the FSOs are based on optical communication. Literature [10] compared the light emitted by the light emitters in multiple wavelength bands by testing multiple bands such as 450,650 , and $890 \mathrm{~nm}$, among which the infrared band $(890 \mathrm{~nm})$ has betteranti-interference and sensitivity [12-13]. In addition, the laser-based FSO communication system has also been studied in depth and several different types of industry modules have been established [11]. However, the main problem with laser FSO is that both the transmitter and the receiver have directivity requirements [14]. Therefore, in practical applications, it is generally necessary to equip the laser FSO device with the telescope orientation device [15], thus restricting the application of the laser FSO.

In previous studies [7-9], FSO showed significant results of energy efficiency by comparison with RF. In addition, one design based on FSO transmission medium was also proposed: the changes in intensity and wavelength emitted by the light emitter are used to define information for the recipient. However, this proposed design still adopts theanalogy system, which is too complex to apply. In other studies [10, 13-14], the lights emitted by multiple light emitters were compared; blue $(450 \mathrm{~nm})$, green (532 $\mathrm{nm})$, red $(650 \mathrm{~nm})$, and infrared $(890 \mathrm{~nm})$ frequencies were tested, finding that the infrared wavelengths have the highest sensitivity and anti-damping performance, but no description about the reliable and simple design were made. Besides, the studies on Laser-based FSO have been conducted [11], [12], [15], but the main problem with lasers is the directivity of light from the emitter to the receiver. Based on previous research, the infrared technology was applied in this paper for WSN communications design.

Therefore, combined with the mutual communication issue between multiple machines in collaborative control of mobile robots, this paper selected infrared communication module to build FSO wireless sensor system. Each node was equipped with eight infrared receiver/transmitter modules for omnidirectional communication. TSAL6200 was selected as the data transmission module, and HS0038B as receiver module.

\section{FSO optical communication system design}

In infrared-based FSO communication systems, the signal modulation, link budget, and bit error rate issues should be mainly considered.

\subsection{ASK Signal Modulation}

The simplest signal technology in the modulation of binary ASK modulation signals can be achieved through the on-off of the normal IO in the embedded system, i.e. OOK. In this modulation, the binary signals 0 and 1 can be used to control GPIO by the "on" and "off" of the switch so that the (infrared) optical communication apparatus is synchronously "closed" and "turned on" to generate the communication signal. The basic infrared ASK modulation process is shown in Fig.1: 


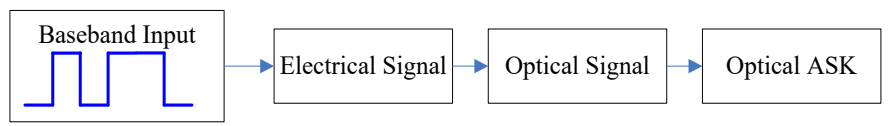

Fig. 1. Optical ASK

ASK signal changes from 0 to 1 in the time range $T b$ can be expressed as:

$$
s(t)\left\{\begin{array}{l}
s l(t)=\frac{1}{\sqrt{1+r_{e}}} A p(t) \cos 2 \pi f_{c} t \\
s 2(t)=\sqrt{\frac{r_{e}}{1+r_{e}}} A p(t) \cos 2 \pi f_{c} t
\end{array}\right.
$$

Assuming $p(t)$ as the initial pulse shape, for the continuous bit stream, the ASK signal can be expressed as:

$$
s(t)=A\left[\sum_{i=-\infty}^{\infty} \frac{d_{i}\left(1-\sqrt{r_{e}}\right)+\sqrt{r_{e}}}{\sqrt{1+r_{e}}} p\left(t-i T_{b}\right)\right] \cos 2 \pi f_{c} t
$$

Where, $d_{i} \in\{0,1\}$ is the $i$-th data bit. When expressed in low-pass equivalent form, formula (2) can be rewritten as:

$$
m(t)=\sum_{i=-\infty}^{\infty} \frac{d_{i}\left(1-\sqrt{r_{e}}\right)+\sqrt{r_{e}}}{\sqrt{1+r_{e}}} p\left(t-i T_{b}\right)
$$

The ASK modulated signal is transmitted in a square wave, so the signal energy of signals 0 and 1 can be expressed as:

$$
\begin{aligned}
& E_{1}=\int_{0}^{T_{b}} s_{1}^{2} d t=\frac{A^{2} T_{b}}{2}\left(\frac{1}{1+r_{e}}\right) \\
& E_{2}=\int_{0}^{T_{b}} s_{2}^{2} d t=\frac{A^{2} T_{b}}{2}\left(\frac{r_{e}}{1+r_{e}}\right)
\end{aligned}
$$

\section{$2.2 \quad$ Link budget}

The link budget method is generally adopted for the WSN node power analysis and estimation. For the standard receiver system, set $P_{r}$ as receiver power, $P_{t}$ transmitter power, $G_{r x}$ receiver gain, $G_{t x}$ transmitter gain, and Loss total loss, then the link budget can be calculatedas [16]:

$$
P_{r}=P_{t}+G_{t x}+G_{r x}-\text { Loss }
$$

One of the biggest problems in optical FSO communication is atmospheric turbulence and the effects of various particulate matter, which can lead to signal loss and 
communication errors. The typical atmospheric attenuation model can be expressed by Beer's law [16]:

$$
t=\exp (-\beta L)
$$

Where, $(\tau)$ is attenuation, $\beta$ is the total attenuation coefficient, and $\mathrm{L}$ is the distance between the transmitter and receiver. $\beta$ can be decomposed as:

$$
\beta=\beta_{\text {abs }} \times \beta_{\text {scatt }}
$$

Where, $\beta_{a b s}$ is the air molecule and aerosol absorption coefficient. Since this parameter is generally small, it can be ignored; $\beta_{\text {scat }}$ is the scattering coefficient of air molecules and aerosols, as shown in Table 1.

Table 1. Scattering coefficients in different media

\begin{tabular}{|l|c|c|}
\hline \multicolumn{1}{|c|}{ Type of Particle } & Radius $(\boldsymbol{\mu m})$ & Size Param X0 \\
\hline Air Molecules & 0.0001 & 0.00074 \\
\hline Haze Particle & $0.01 \sim 1$ & $0.074 \sim 7.4$ \\
\hline Fog Droplets & $1 \sim 20$ & $7.4 \sim 147.8$ \\
\hline Rain Droplets & $100 \sim 1000$ & $740 \sim 7400$ \\
\hline Snow Flakes & $1000 \sim 5000$ & $7400 \sim 37000$ \\
\hline
\end{tabular}

At the transmitting terminal, the typical pulse of TSAL6200 is $20 \mathrm{~ms}$. At this time, the energy consumption is $25 \mathrm{~mW} /$ spherical radiant intensity. The pulse waveform is shown in Fig.2.

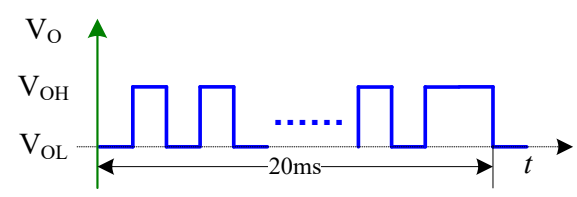

Fig. 2. 20ms time waveform

At the receiving terminal, the additional amplifiers (such as Fresnel lenses, etc.), the gain on the transmitter and receiver are all ignored; the minimum received power of the HS0038B device is $0.08 \mathrm{~mW} / \mathrm{m}^{2}$. Then, $G_{t x}=G_{r x}=0$. Therefore, the parameter Gaincan be ignored. The link budget calculation can be simplified as:

$$
P_{r}=P_{t}-[\exp (-\beta L) \times L]
$$

Assume that in the analysis and experiment, all the above parameters are constant, and by querying table 1 , substitute the air attenuation coefficient into formula (7), then, the link budget analysis in this study will be turned into:

$$
P_{r}=25 m W-[\exp (-0.00074 \times L) \times L]
$$




\subsection{BER Performance Analysis}

In this paper, the bit error rate (BER) was used to analyse the error probability of each symbol bit. BER can be expressed as

$$
P e c \mid=\frac{P(e \mid 1)}{2}+\frac{P(e \mid 0)}{2}
$$

Where, $P e c$ is the conditional error probability, $\mathrm{P}(\mathrm{e} \mid 0)$ is the probability that the received signal is the logic 1 , and the transmitted signal is actually the logic $0 ; \mathrm{P}(\mathrm{e} \mid 1)$ is the probability that the received signal is the logic 0 and the transmitted signal is actually the logic 1 . In this experiment, a set of fixed pulses was used for testing and the rising edge trigger was used. Therefore, the BER can be expressed as:

$$
B E R=P e=0.5\left[P_{0}\left(n>n_{t h}\right)+P_{1}\left(n<n_{t h}\right)\right]
$$

It can be seen that $B E R$ is half of the sum of the error probabilities on the logic $0\left(P_{0}\right)$, because the received voltage level is greater than the receiver voltage threshold $\left(n_{t h}\right)$, and there is an error probability on logic $1\left(P_{t}\right)$ when the received voltage level is less than the receiver voltage threshold.

\section{System design}

\subsection{Overall system framework design}

The purpose of this study is to build one FSO-based WSN communication system for mobile robots and support collaborative control. Therefore, the entire system mainly consists of hardware subsystem and software subsystem. For the hardware subsystem, AT Mege2560 (Arduino 2560) was selected as the embedded master controller, while integrating the sensors, controllers and communication components. The system hardware framework is shown in Fig.3.

The transmitter terminal includes IC555 modulation circuit and eight TSAL 6200 infrared transmitters modules. The receiver terminal consists of an M74HC30B1 Nand Gate and eight HS0038B infrared receivers. Fig.4 depicts the system launching and receiving circuit. 


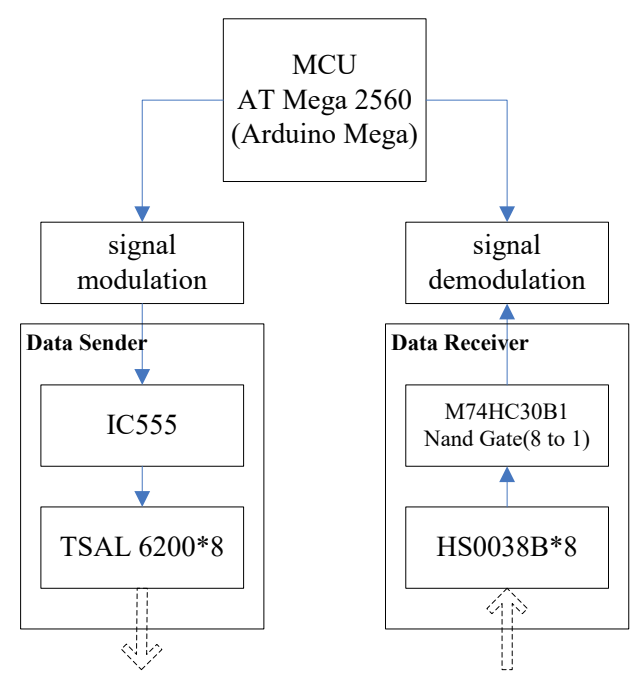

Fig. 3. System hardware block diagram

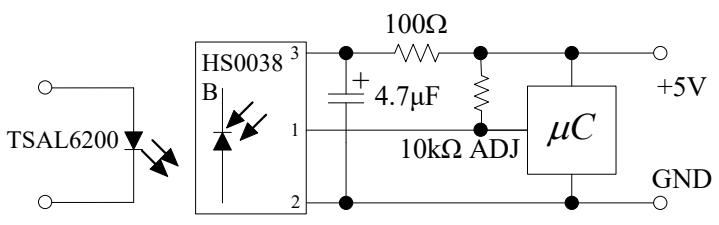

Fig. 4. Functional block diagram of transmitting and receiving circuit

\subsection{Infrared data transmission subsystem design}

The data transmission unit TSAL6200 operates at a wavelength of 940nm and is compatible with a variety of infrared receivers. The technical specifications are shown in Table 2.

Table 2. TSAL 6200 data sheet

\begin{tabular}{|l|c|c|}
\hline \multicolumn{1}{|c|}{ Params } & Test Condition & Typical Value \\
\hline Forward Voltage & $\mathrm{I}_{\mathrm{F}}=100 \mathrm{~mA} \mathrm{~T} T_{\mathrm{P}}=20 \mathrm{mS}$ & $1.35 \mathrm{~V}$ \\
\hline Radian Intensity & $\mathrm{I}_{\mathrm{F}}=100 \mathrm{~mA} \mathrm{~T} \mathrm{~T}_{\mathrm{P}}=20 \mathrm{mS}$ & $25 \mathrm{~mW}$ \\
\hline Radian Power & $\mathrm{I}_{\mathrm{F}}=100 \mathrm{~mA} \mathrm{~T} \mathrm{~T}_{\mathrm{P}}=20 \mathrm{mS}$ & $35 \mathrm{~mW}$ \\
\hline Angle of Half Intensity & $\mathrm{N} / \mathrm{A}$ & $\pm 30^{\circ}$ \\
\hline Peak Wavelength & $\mathrm{I}_{\mathrm{F}}=100 \mathrm{~mA}$ & $940 \mathrm{mn}$ \\
\hline
\end{tabular}

The NE555 timer was used for ASK signal modulation. In this paper, the $38 \mathrm{KHz}$ signal was mainly modulated in an unsteady manner, and data was transmitted through the TSAL6200. 


\subsection{Infrared data receiver subsystem design}

The data receiving unit HS0038B operates at the frequency is $38 \mathrm{KHz}$. It is used with TSAL. Its technical specifications are shown in Table 3:

Table 3. HS0038B data sheet

\begin{tabular}{|l|c|c|}
\hline \multicolumn{1}{|c|}{ Params } & Test Condition & Typical Value \\
\hline Supply Voltage & $\mathrm{E}_{\mathrm{v}}=0, \mathrm{~V}_{\mathrm{s}}=3.3 \mathrm{~V}$ & $2.5 \sim 5.5 \mathrm{~V}$ \\
\hline SupplyCurrent & $\mathrm{N} / \mathrm{A}$ & $0.35 \mathrm{~mA}$ \\
\hline Power Consumption & $\mathrm{T}$ Ambient $\leq 85^{\circ}$ & $10 \mathrm{~mW}$ \\
\hline Transmission Distances & $\mathrm{E}_{\mathrm{v}}=0$, IR TSAL 6200 & $35 \mathrm{~m}$ \\
\hline Minimum Irradiance & N/A & $0.08 \mathrm{~mW} / \mathrm{m}^{2}$ \\
\hline Directivity & Angle of half transmission distances & $\pm 45^{\circ}$ \\
\hline
\end{tabular}

\subsection{Prototype node design}

In order to enable the mobile robot to receive signals omnidirectionally in the horizontal direction, eight infrared transmitters and receivers were respectively deployed with $45^{\circ}$ angular difference. The infrared receiving device was placed behind the transmitting device to avoid emission interference. The prototype node system is shown in Fig.5.

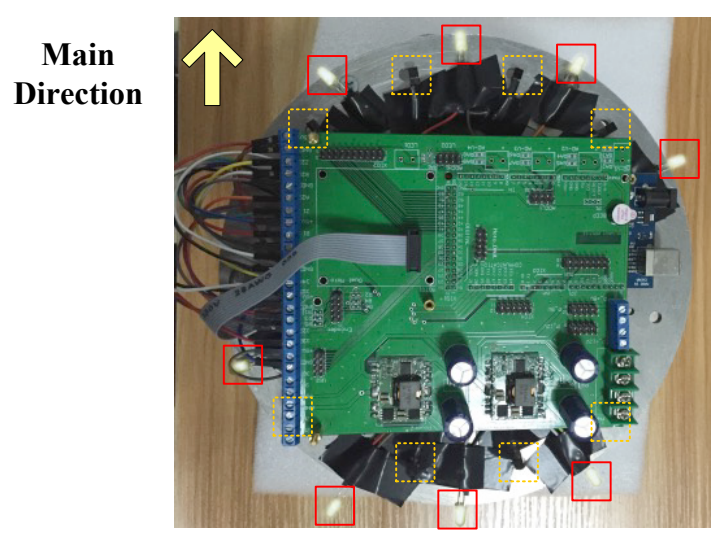

Fig. 5. Prototype node system

The M2560 MCU is not only responsible for data acquisition and motion control of mobile robot sensors, but also for communicating with other robots and upper-level control systems. When information interaction (mainly attitude and position data) is required between them, the NE555 can be used to modulate the signal through the $38 \mathrm{KHz}$ square wave. The modulated signal is sent out through the 8-channel TSAL 6200 at the same time, covering the entire range at 360-degree (because the robot can only operate on the same plane [17]). The system transmission process is shown in Fig.6. 


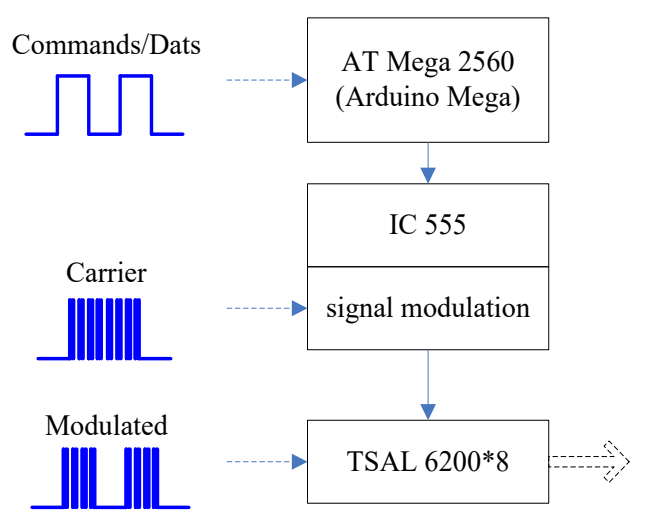

Fig. 6. Transmitter system framework

For the receiving part, $8 \mathrm{HS} 0038 \mathrm{~B}$ can ensure to capture the information sent by other robots in any horizontal direction. Because of the information sent by multiple sending modules (possibly from different robots), the M74HC30B1 NAND Gate was used for signal processing.

The reception of ASK signals depends heavily on the performance of the HS0038B because these devices have been already equipped with a series of band pass and demodulators that replace the mathematical functions of the ASK receiver. In this study, there were 8 receivers on each device node, i.e., there were 8 devices HS0038B on each cell of the node. The NAND Gate is used to simultaneously process the information from the 8 receivers, e.g., two signals are detected at some time by the system (Channel1 and Channel8); these signals will be logically inverted at HS0038B, so, the above NAND Gate needs to be used for signal inversion to restore the original signal state. Fig. 7 shows the reception process.

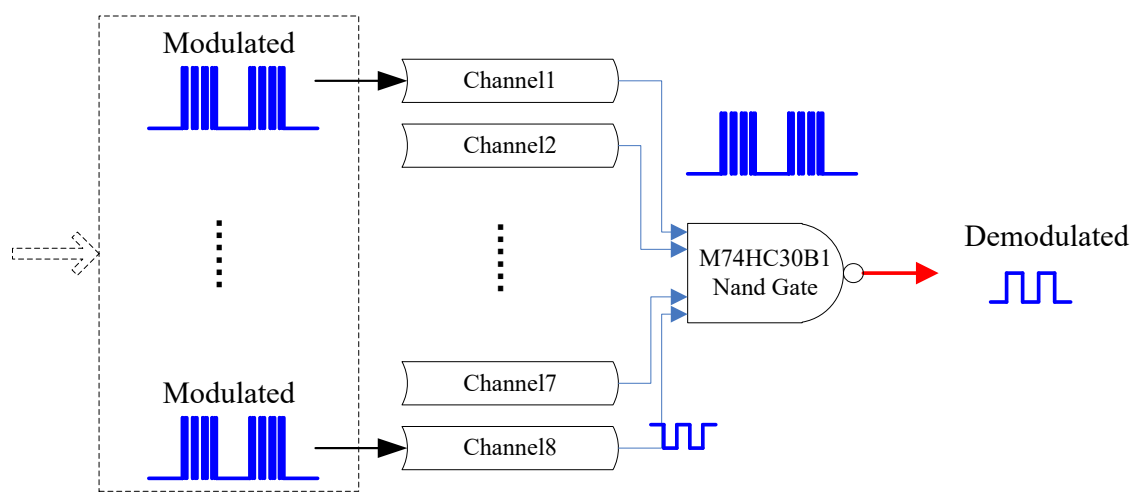

Fig. 7. Receiver system framework 


\subsection{Software subsystem design}

The software subsystem corresponds to the hardware subsystem. It is also divided into the transmitting process and receiving process. The algorithm flow of the transmitter module is shown in Fig.8.

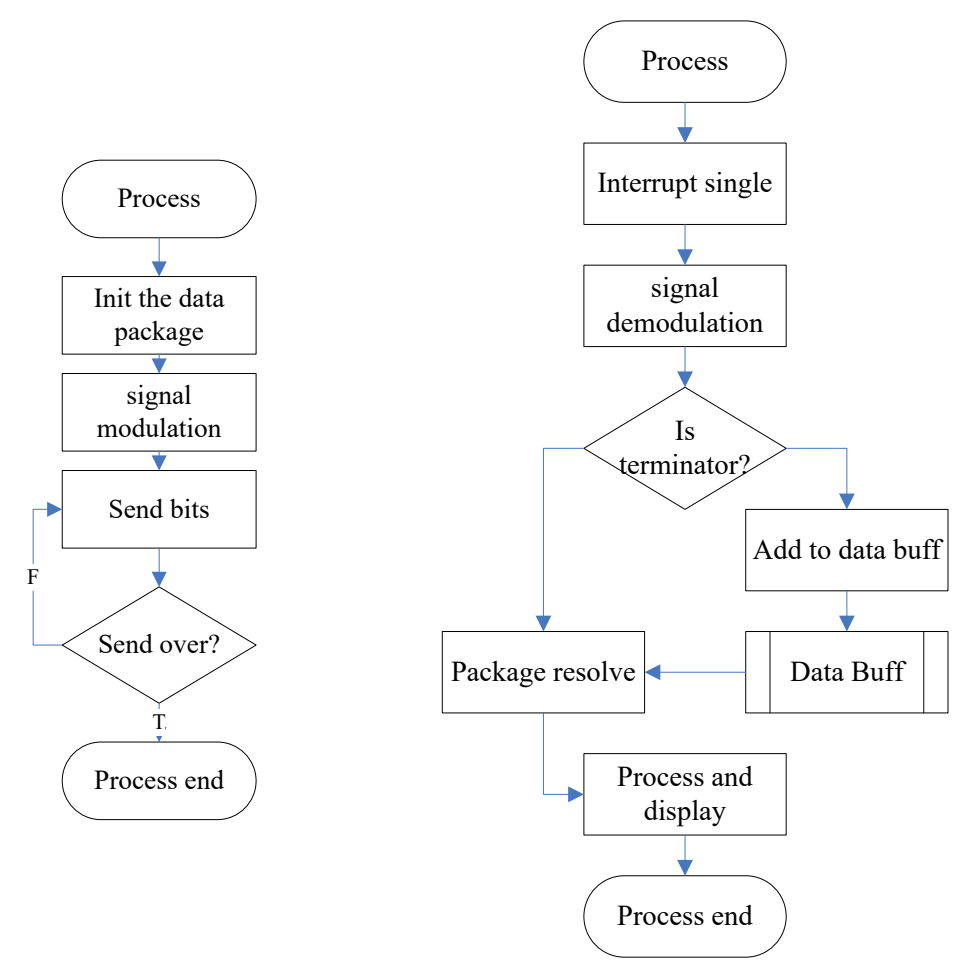

Fig. 8. Flow chart of transmitter task

Fig. 9. Receiver software processing flow chart

Although bit streams were used in the tests, there was a "stop bit" or "terminator" at the control layer. The sending process was completed when the terminator was detected. At the receiving terminal the signal is captured using the rising edge trigger, so that no matter whether it is a normal pulse or interference, it will be captured. The transmitting process is shown in Fig.9.

\section{$4 \quad$ Test and results analysis}

\subsection{BER analysis and measurement}

In order to test the power consumption, coverage and bit error rate (BER) of the designed infrared FSO communication system, two nodes were used for testing. Fig.10 shows the test signal method. 


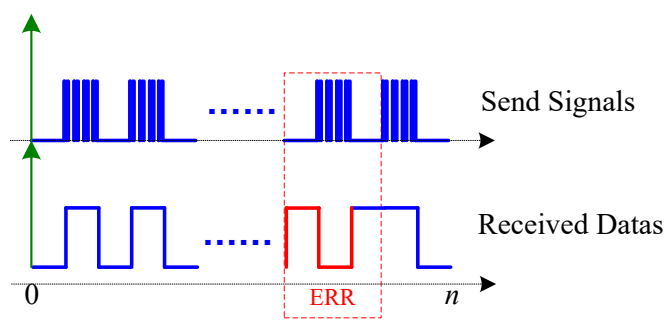

Fig. 10.Comparison between test signal and actual output of receiver HS0038B

In the retransmission test of the sending node, 100 sets of pulses were fixedly transmitted, and the receiving node counted the detected rising edge pulses. The location of the sending node was fixed, and the receiving node gradually moved away from the transmitting node during the test at a distance of $1 \mathrm{~m}$ each time. The test result is shown in Fig.11:

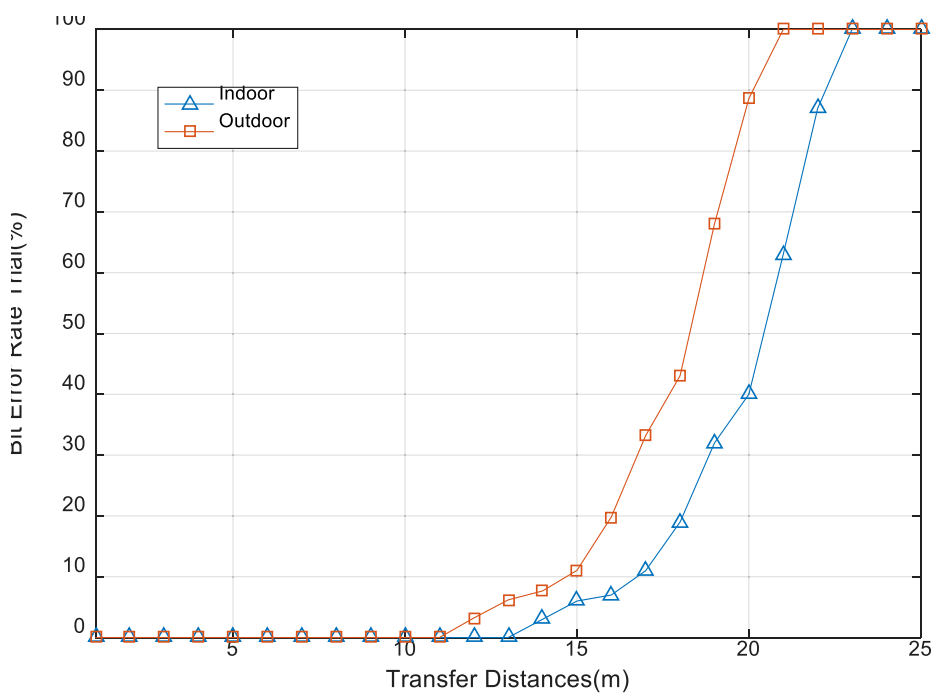

Fig. 11.Test results: BER vs. test distance

The formula (12) was used for BER analysis. At the distance $<14 \mathrm{~m}$, the communication quality between the nodes is good, and the test $\mathrm{BER}=0 \%$. But at the distance $>14 \mathrm{~m}$, the BER began to rise rapidly. When it reaches $22 \mathrm{~m}, \mathrm{BER}=100 \%$, it cannot communicate normally.

\subsection{Link budget analysis based on data table}

The formula (10) was used to analyse the link budget. Fig.12 shows the results. 


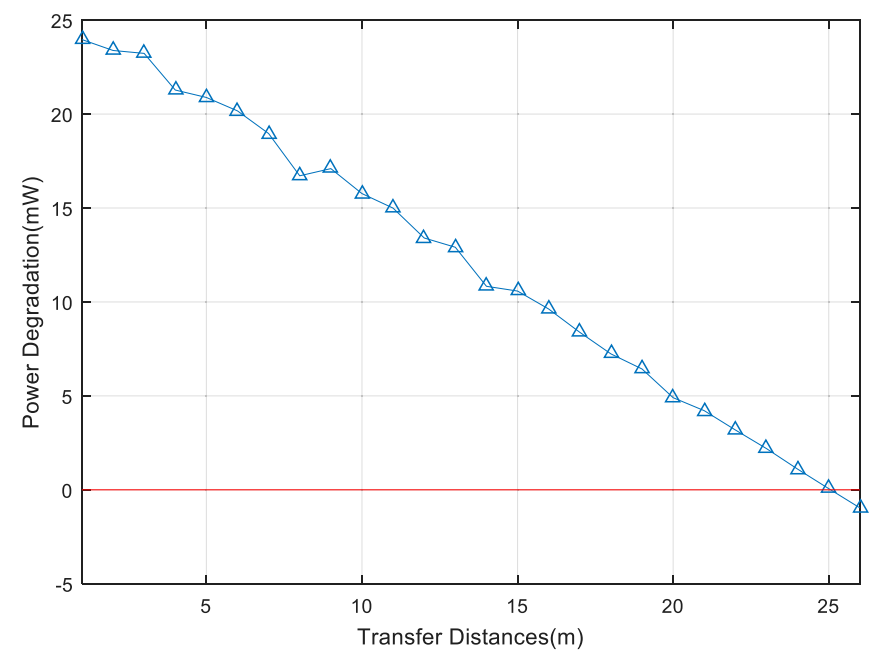

Fig. 12. Test results: receiver power vs. distance

From Fig.12, it can be seen that when the distance between nodes is about 25 meters, the received power is zero, that is, the limit receiving distance is 25 meters. This is quite different from the actual communication distance of 14 meters shown in Fig.12, because the rising edge trigger requires a sufficient level signal (power signal), which can be further analysed by comparing the pulse shape with the BER.

\subsection{Comparison between BER and pulse waveform}

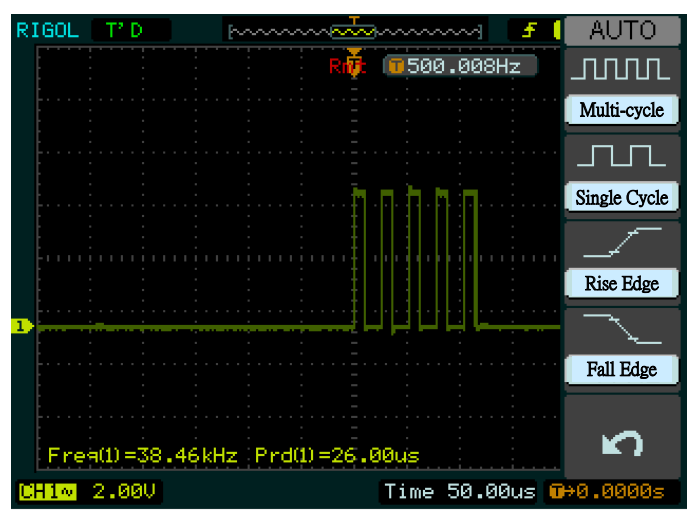

Fig. 13.Distance: $1 \sim 14$

The oscilloscope was used to observe the transmitted signal and then count the BER. The oscilloscope is RIGOL DS1102D with the sampling frequency of $100 \mathrm{MHz}$ and sampling depth of $1 \mathrm{GSa} / \mathrm{s}$. Because the infrared communication frequency is $38 \mathrm{KHz}$, the sampling time is Time $=50 \mu$ s and the rising edge is used to trigger the capture. In 
order to test the BER, the signal of the same group was sent periodically. The format of the signal is $s=101010101$, where the first 8 bits are data bits and the last bit is the check bit. Fig. 13 shows the ideal waveform.

The measurement distances were changed separately, and then the results of the signal were captured by the oscilloscope (Fig.13- Fig.15).

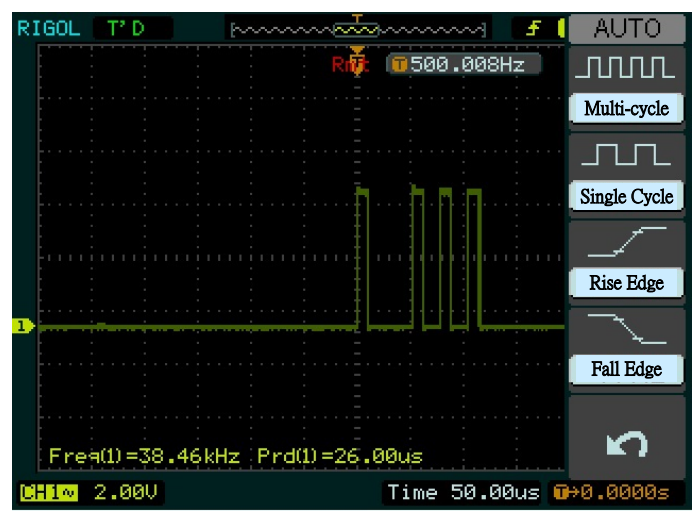

Fig. 14.Distance:14 17

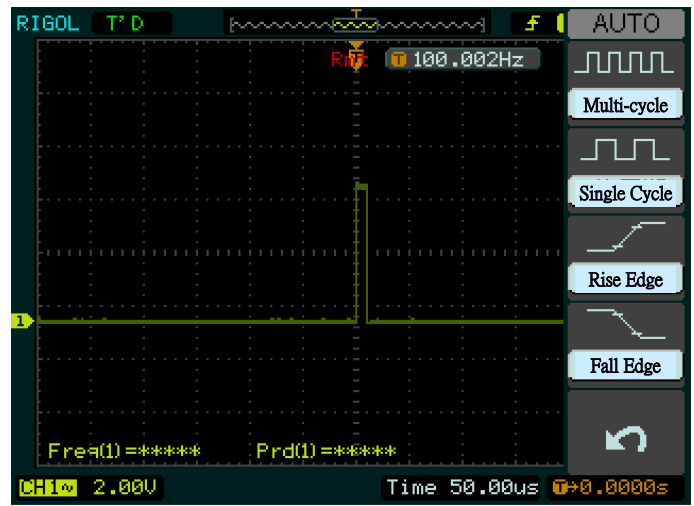

Fig. 15.Distance:17 22

It can be seen that as the distance increases, the number of signals to be captured gradually decreases. At the distances $>22 \mathrm{~m}$, no signal can be captured.

\section{Conclusions}

In this paper, an infrared-based FSO communication system was designed under the application background of robotic cooperative control. Each node used 8 channels of TSAL6200 as the transmitter module and 8 channels of HS0038B as the receiver module to achieve horizontal plane omnidirectional communication. This can reduce the 
communication system power consumption and solve the FSO directional communication problem.

Test results show that the system can perform effective continuous communication within 14 meters, when the average BER is $0 \%$; the maximum communication distance is 22 meters; the maximum effective communication distance is about 18 meters for the acceptable BER $(<30 \%)$. Although the theoretical communication distance can reach 35 meters, due to the influence of circuit configuration and atmospheric turbulence etc., the actual communication distance is only $51 \%$ of that in the data sheet, which still has great potential for improvement.

\section{Acknowledgement}

Public Welfare Scientific Research Program is funded by the Hubei Provincial Department of Education (B2017503). Scientific research project is funded by Wenzhou City (Grant No. LY G20160020).

\section{$7 \quad$ Reference}

[1] Liu, J., Xiong, K., Fan, P., Zhong, Z. (2017).RF Energy Harvesting Wireless Powered Sensor Networks for Smart Cities. IEEE Access, 5: 9348-9358. https://doi.org/10.1109/ACC ESS.2017.2703847

[2] Wang, P., Zhang, J., Guo, L., Shang, T., Cao, T. (2017). Performance Analysis for RelayAided Multihop BPPM FSO Communication System Over Exponentiated Weibull Fading https://doi.org/10.1109/JPHOT.2015.2445765

[3] Alzenad, M., Shakir, Z., Yanikomeroglu, H., Alouini, M.S. (2018). FSO-based Vertical Backhaul Fronthaul Framework for 5G+ Wireless Networks. IEEE Communications Magazine, 6(1): 218-224. Bhatnagar, M.R., Ghassemlooy, Z. (2016). Performance Analysis of Gamma-Gamma Fading FSO MIMO Links with Pointing Errors. Journal of Light wave Technology, 34(9): 2158-2169. https://doi.org/10.1109/JLT.2016.2526053

[4] Ahdi, F., Subramaniam, S. (2016). Capacity enhancement of RF wireless mesh net-works through FSO links. IEEE/OSA Journal of Optical Communications \& Net-working, 8(7): 495-506. https://doi.org/10.1364/JOCN.8.000495

[5] Ismail, T., Leitgeb, E. (2016). Performance analysis of SIM-DPSK FSO system over lognormal fading with pointing errors. International Conference on Transparent Opti-cal Networks, 1-4. https://doi.org/10.1109/ICTON.2016.7550350

[6] Gappmair, W., Nistazakis, H. (2017). Subcarrier PSK Performance in Terrestrial FSO Links Impaired by Gamma-Gamma Fading, Pointing Errors, and Phase Noise. Journal of Lightwave Technology, 35(9), 1624-1632. https://doi.org/10.1109/JLT.2017.2685678

[7] Selvi, M., Logambigai, R., Ganapathy, S., Nehemiah, H.K., Arputharaj, K. (2017). An Intelligent Agent and FSO Based Efficient Routing Algorithm for Wireless Sensor Network. International Conference on Recent Trends \& Challenges in Computational Models, 100105. https://doi.org/10.1109/ICRTCCM.2017.43

[8] Zhang, N. (2017). An Algorithm of Wireless Sensor Network Based on Free Space Op-tical Communication. International Journal of Online Engineering, 13(7): 108-119. https://doi.org/10.3991/ijoe.v13i07.7292 
[9] Turán, J., L'uboš, O. (2016). Experimental FSO network availability estimation using interactive fog condition monitoring. Proceedings of the Spie, 142: 1014223. https://doi.org/10.1117/12.2263614

[10] Solanki, S., Kohli, J. (2016). Wireless sensor network survey. Far East Journal of Electronics \& Communications, 767-776. https://doi.org/10.17654/ECSV3PII16767

[11] Makki, B., Svensson, T., Eriksson, T., Alouini, M.S. (2016). On the Performance of RFFSO Links with and Without Hybrid ARQ. IEEE Transactions on Wireless Commu-nications, 15(7): 4928-4943. https://doi.org/10.1109/TWC.2016.2549537

[12] Anguita, D., Brizzolara, D., Parodi, G. (2009). Optical Wireless Communication for Underwater Wireless Sensor Networks: Hardware Modules and Circuits Design and Implementation. IEEE Conference Publication on OCEANS, 1-2. $\leq<$ https://doi.org/10.1109/OCEANS. 2010.5664321

[13] Ghelardoni, L., Ghio, A., Anguita, D. (2012). Smart Underwater Wireless Sensor Networks. Electrical \& Electronics Engineers in Israel, 23(99): 1-5. https://doi.org/10.1109/EEEI.2012.6376941

[14] Anand, A., Aarthi, G., Vardhan, H. (2015). Improving the efficiency of FSO communi-cation system with multi-laser beams. International Journal of Applied Engineering Research, 10(12): 32653-32662

[15] Alkholidi, A.G., Altowij, K.S. (2014). Free Space Optical Communication-Practice and theory. INTECH, 16(4): 2231-2258. https://doi.org/10.5772/58884

[16] Dutta, P., Kumar, A. (2017). Intelligent calibration technique using optimized fuzzy logic controller for ultrasonic flow sensor. Mathematical Modelling of Engineering Problems, 4(2): 91-94. https://doi.org/10.18280/mmep.040205

\section{Authors}

Jinyong Huang was born in March 1981, Xiaogan city, Hubei province, received a master's degree in June 2014. His research field is computer network application technology. Graduated from Wuhan University, Wuhan, China. He is working in the College of Technology, Hubei Engineering University, Xiaogan 432000, China.

Zhe Li received the Ph.D. degree. He is currently a Lecturer with the School of Economics and Management, Hubei Engineering University, Xiaogan 432000, China. His research interests include the consistency control of multiple agents in complex environments, multi-rates with multi-agent systems, and distributed control systems.

Article submitted 05 September 2018. Resubmitted 14 October 2101. Final acceptance 16 October 2018. Final version published as submitted by the authors. 